\title{
MATHEMATICAL MODELS OF CALCIUM AND TIGHT JUNCTIONS IN NORMAL AND RECONSTRUCTED EPIDERMIS
}

\author{
MATTHEW P. ADAMS
}

(Received 2 November 2015; first published online 11 January 2016)

2010 Mathematics subject classification: primary 92B05; secondary 35A99.

Keywords and phrases: calcium profile, epidermis, mathematical biology, skin substitute, tight junctions.

The epidermis is the uppermost layer of the skin. It possesses a distinct stratified structure consisting of four sublayers and a characteristic calcium profile which is strongly implicated in regulating this structure. The calcium concentration rises from low concentrations in the lowest sublayers (stratum basale and stratum spinosum) to a peak in the overlying sublayer (stratum granulosum) and then drops to negligible levels in the most superficial sublayer (stratum corneum). The factors that regulate the formation of the epidermal calcium profile are currently a source of debate. These factors definitely include (1) passive regulation by the primary barrier function of the stratum corneum, but may also include (2) active regulation due to calcium exchanges between cells and the surrounding extracellular fluid (ECF) and (3) extracellular transport limitation in the stratum granulosum due to the secondary barrier formed by tight junctions there. The relative importance of the latter two factors to the formation of the epidermal calcium profile has yet to be resolved.

Epidermal cells (keratinocytes) can also be cultured in vitro to form a multilayered structure ('reconstructed epidermis') that strongly resembles its in vivo counterpart ('normal epidermis'). However, reconstructed epidermis typically lasts less than a month, following a well-defined but unexplained growth and deterioration pattern. This is a serious clinical issue that affects its ability to be stored as a readily available skin replacement for patients requiring emergency treatment. Because the calcium distribution regulates epidermal structure, it is quite possible that its altered dynamics in vitro could be responsible for the deterioration of reconstructed epidermis, although this has not yet been investigated.

Thesis submitted to Queensland University of Technology in January 2013; degree approved on 19 February 2015; supervisors Dann Mallet and Graeme Pettet.

(c) 2016 Australian Mathematical Publishing Association Inc. 0004-9727/2016 \$16.00 
This thesis examines both normal and reconstructed epidermis using multiphase models consisting of two phases (cells and fluid), in order to achieve two major objectives: (1) determine the key mechanisms that regulate the calcium profile in unwounded normal epidermis and (2) identify the possible contributors to the growth and deterioration pattern of reconstructed epidermis. Our application of multiphase models to normal and reconstructed epidermis is novel and has not yet been reported elsewhere.

For the first objective, it is already well established that the epidermal calcium profile is passively regulated by the presence of stratum corneum. We assumed that this was true and thus first investigated the spatial variation of calcium exchange through the viable epidermal sublayers using the steady state solution of a multiphase model parameterised for both human and murine epidermis [1]. To accomplish this, velocity profiles for the cells and ECF were calculated from experimental data for the proliferation and growth kinetics of keratinocytes in these sublayers. We assumed that the diffusion of calcium through the ECF could be approximated by its diffusion in water at skin temperature. From this diffusion consideration and the calculated ECF velocity profiles, the relative contributions of advection and diffusion to the motion of extracellular calcium were compared. We found that diffusion governs extracellular calcium motion. This indicated that the intracellular calcium was the main source of the total epidermal calcium profile. Using the equations of the multiphase model at steady state together with some reasonable assumptions, we used the calculated cell velocity profiles and experimental data for the total calcium profiles to predict the spatial profile of calcium exchange between cells and the ECF in the viable epidermis. We found that there is net influx of calcium ions into keratinocytes from the ECF in the lowest epidermal sublayers (stratum basale and stratum granulosum) and that there is net outflux of these ions in the stratum granulosum. This indicated that the spatial pattern of calcium exchange between keratinocytes and ECF is an important contributor to the regulation of the calcium profile in unwounded epidermis.

We then updated this steady state multiphase model to include the presence of tight junctions in the stratum granulosum [4]. We found that considering tight junctions as a local reduction in the diffusion coefficient could explain the remaining discrepancy between our model and the experimental data for the epidermal calcium profile, the latter showing a rise in extracellular calcium concentration in the stratum granulosum. We concluded that the calcium profile in unwounded epidermis most likely forms in response to all three investigated factors: the barrier functions of the stratum corneum and tight junctions, and the spatial pattern of calcium exchange between the keratinocytes and ECF.

For the second objective, we developed a spatiotemporal multiphase model of the growth of reconstructed human epidermis, consisting of partial differential equations that describe the distributions of intracellular and extracellular calcium, tight junctions and a signal chemical hypothesised to induce tight junction formation, and ordinary differential equations that describe the motion of the epidermal sublayer boundaries and hence the growth of reconstructed epidermis [2]. The model considered the growth 
of reconstructed epidermis from basal keratinocytes to a fully stratified structure in three temporal stages, each stage corresponding to the formation of individual suprabasal layers (stratum spinosum, stratum granulosum and stratum corneum).

The model was solved using three different methods [3]. First, an analytical solution of the spatiotemporal dynamics could be derived for certain parameter values. Second, steady states of the model were calculated using a root-finding algorithm, which numerically calculated the steady state distributions of intracellular and extracellular calcium, signal chemical and tight junctions for guessed values of the sublayer boundary positions, and then iteratively updated the guesses of these boundary positions based on the mismatch between these guesses and their expected positions according to their governing ordinary differential equations. Third, the full spatiotemporal model was solved numerically by first employing a mathematical transformation of the growing spatial domain to a fixed domain and then numerically solving the resulting system of ordinary and partial differential equations. The model demonstrated that epidermal skin substitute deterioration cannot be attributed to in vitro dysfunction of tight junction expression in the stratum granulosum, but some deterioration is possible if the extracellular diffusion of calcium throughout the reconstructed epidermis is reduced from reported values in the literature. Hence, the reason for the growth and deterioration of reconstructed epidermis remains an open question, but the mathematical modelling and conceptual background developed here may aid future efforts to resolve this question.

The outcomes of this research and additional information can be found in [1-4].

\section{References}

[1] M. P. Adams, D. G. Mallet and G. J. Pettet, 'Active regulation of the epidermal calcium profile', J. Theoret. Biol. 301 (2012), 112-121.

[2] M. P. Adams, D. G. Mallet and G. J. Pettet, 'A continuum model of the growth of engineered epidermal skin substitutes', ANZIAM J. Electron. Suppl. 53 (2012), C90-C109.

[3] M. P. Adams, D. G. Mallet and G. J. Pettet, 'Solution methods for advection-diffusion-reaction equations on growing domains and subdomains, with application to modelling skin substitutes', ICCM2012, 4th Int. Conf. Computational Methods, Gold Coast, Australia, 25-28 November 2012, 230.

[4] M. P. Adams, D. G. Mallet and G. J. Pettet, 'Towards a quantitative theory of epidermal calcium profile formation in unwounded skin', PLoS One 10 (2015), e0116751.

MATTHEW P. ADAMS, Mathematical Sciences School, Queensland University of Technology, Brisbane, Australia e-mail:m.adams5@uq.edu.au 